\title{
Clinical laboratories collaborate to resolve differences in variant interpretations submitted to ClinVar
}

\author{
Steven M. Harrison, $\mathrm{PhD}^{1,2}$, Jill S. Dolinsky, $\mathrm{MS}^{3}$, Amy E. Knight Johnson, $\mathrm{MS}^{4}$, \\ Tina Pesaran, MA, MS³, Danielle R. Azzariti, MS1', Sherri Bale, PhD ${ }^{5}$, Elizabeth C. Chao, MD ${ }^{3,6}$, \\ Soma Das, PhD ${ }^{4}$, Lisa Vincent, $\mathrm{PhD}^{5}$ and Heidi L. Rehm, $\mathrm{PhD}^{1,2,7,8}$
}

\begin{abstract}
Purpose: Data sharing through ClinVar offers a unique opportunity to identify interpretation differences between laboratories. As part of a ClinGen initiative, four clinical laboratories (Ambry, GeneDx, Partners Healthcare Laboratory for Molecular Medicine, and University of Chicago Genetic Services Laboratory) collaborated to identify the basis of interpretation differences and to investigate if data sharing and reassessment resolve interpretation differences by analyzing a subset of variants.
\end{abstract}

Methods: ClinVar variants with submissions from at least two of the four participating laboratories were compared. For a subset of identified differences, laboratories documented the basis for discordance, shared internal data, independently reassessed with the American College of Medical Genetics and Genomics-Association for Molecular Pathology (ACMG-AMP) guidelines, and then compared interpretations.

\section{INTRODUCTION}

Interpreting the clinical significance of genetic variants is a challenging process that involves gathering and assessing available evidence, followed by formal classification based on this evidence. Variant classifications from laboratories may confirm the cause of disease, illuminate an etiology not previously considered, or guide treatment decisions. Historically, variant classifications from clinical laboratories have mostly been unavailable to the larger genetics community, except for the small fraction published in journals. Recent efforts by the National Institutes of Health-funded Clinical Genome Resource (ClinGen; https://www.clinicalgenome.org/) to support widespread data sharing using the ClinVar database have accelerated the public sharing of interpreted variants, particularly by clinical laboratories. ${ }^{1}$ The ClinVar database (http:// www.ncbi.nlm.nih.gov/clinvar/), maintained by the National Center for Biotechnology Information, archives and aggregates
Results: At least two of the participating laboratories interpreted 6,169 variants in ClinVar, of which $88.3 \%$ were initially concordant. Laboratories reassessed 242/724 initially discordant variants, of which $87.2 \%$ (211) were resolved by reassessment with current criteria and/or internal data sharing; $12.8 \%$ (31) of reassessed variants remained discordant owing to differences in the application of the ACMG-AMP guidelines.

Conclusion: Participatinglaboratories increased their overall concordance from 88.3 to $91.7 \%$, indicating that sharing variant interpretations in ClinVar-thereby allowing identification of differences and motivation to resolve those differences-is critical to moving toward more consistent variant interpretations.

Genet Med advance online publication 16 March 2017

Key Words: ACMG-AMP guidelines; ClinVar; data sharing; variant interpretation

submitted interpretations of the clinical significance of variants, with opportunities for each submitter to provide supporting evidence, and indicates whether the submitted interpretations are concordant or discordant. ${ }^{2}$ Sharing variants in ClinVar allows for crowd-sourcing the enormous labor of variant interpretation and provides transparency and inherent "peer review" of variant classifications. Additionally, sharing data facilitates identification of differences in variant interpretation, providing a valuable opportunity for clinical laboratories and other ClinVar submitters to resolve those differences.

Prior reports of inconsistencies in variant interpretations have ranged from $53 \%$ discordance in a double review of pathogenic or likely pathogenic variants ${ }^{3}$ to $53 \%$ discordance of uncertain significance interpretations from one clinical laboratory compared to another ${ }^{4}$ and $60 \%$ discordance of a single laboratory's interpretations compared to those of other clinical laboratories. ${ }^{5}$ However, these comparisons probably overestimate discordance because most compare a current interpretation

\footnotetext{
${ }^{1}$ Laboratory for Molecular Medicine, Partners HealthCare Personalized Medicine, Cambridge, Massachusetts, USA; ${ }^{2}$ Harvard Medical School, Boston, Massachusetts, USA; ${ }^{3}$ Ambry Genetics, Aliso Viejo, California, USA; ${ }^{4}$ Department of Human Genetics, The University of Chicago, Chicago, Illinois, USA; ${ }^{5}$ GeneDx, Gaithersburg, Maryland, USA; ${ }^{6}$ Department of Pediatrics, Division of Genetics and Genomics, University of California Irvine, Irvine, California, USA; ${ }^{7}$ Department of Pathology, Brigham \& Women's Hospital, Boston, Massachusetts, USA; ${ }^{8}$ The Broad Institute of MIT and Harvard, Cambridge, Massachusetts, USA. Correspondence: Steven M. Harrison (sharrison6@partners.org) 
to historic interpretations from other source(s), which may be outdated and not based on an assessment of the same evidence. Reported discordant counts are typically limited to oneor two-step differences between the three major classification levels: "pathogenic (P)/likely pathogenic (LP)," "variants of uncertain significance (VUS)," and "likely benign (LB)/benign (B)." Differences in confidence or "likelihood" are not included in these discordant counts because they are unlikely to impact clinical care and are not reported as conflicts in ClinVar. ${ }^{2,7}$ Differences in variant interpretations between clinical laboratories often occur as a result of differences in algorithms for weighting evidence to reach a classification or differences in available evidence, such as internal frequency and case data or access to external proprietary databases. A recent report found that $33 \%$ of interpretation differences between clinical laboratories were due to a lack of access to privately held data. ${ }^{5}$

Historically, clinical laboratories developed their own internal methods for variant assessment and classification, and some relied heavily on claims in the peer-reviewed literature; both contributed to inconsistencies in variant classification between laboratories. In 2015, the American College of Medical Genetics and Genomics (ACMG) and the Association for Molecular Pathology (AMP) published a joint guideline for variant interpretation that provides an evidence-based framework for classifying variants, requiring laboratories to critically review literature claims and base classifications on a review of the primary evidence. ${ }^{8}$ By including a defined set of evidence types, the ACMG-AMP guidelines enable tracking of the specific evidence types and strengths used to classify each variant, which facilitates classification rationale transparency and easier classification comparisons. However, because of the complexity of variant interpretation, application of the criteria still requires subjective interpretation. A recent study piloting the 2015 ACMG-AMP guidelines across nine sites involved in the Clinical Sequencing Exploratory Research (CSER) program $^{9}$ found that classifications were initially $43.4 \%(43 / 99)$ discordant but resolution was possible for $67.4 \%$ (29/43) of initial interpretation differences, leaving only 5\% (5/99) of variants with medically significant differences (P/LP versus VUS/ $\mathrm{LB} / \mathrm{B})$ remaining at the conclusion of the study. ${ }^{7}$ This exercise, however, was performed before laboratories had operationally implemented the guidelines and, for the majority of variants, was not based on variants encountered during routine clinical testing. In addition, in May 2015, ClinVar was found to have $17 \%$ discordance in variants with multiple submitters, but this discordance is also based on all interpretations submitted to ClinVar, including interpretations from research laboratories, locus-specific databases, and aggregate databases (Online Mendelian Inheritance in Man (OMIM) and GeneReviews). ${ }^{1}$ Therefore, we sought to examine variant interpretation concordance in the routine clinical laboratory environment.

Four clinical laboratories-Ambry Genetics, GeneDx, Partners HealthCare Laboratory for Molecular Medicine (LMM), and University of Chicago Genetic Services Laboratories (UCGSL)-are working together to identify interpretation differences after submission to ClinVar and collaborating to resolve these differences by data sharing and reassessment with laboratories' current criteria, consistent with the ACMG-AMP guidelines. Although complete concordance in interpretations is unlikely owing to subjectivity in weighting evidence and interpretation of guidelines, our goal was to track detailed data for a subset of all interpretation differences to identify trends regarding the basis of interpretation differences between laboratories and to investigate the ability of laboratories to resolve differences in variants already clinically reported, which will educate the community on how to move toward more consistent variant interpretations.

\section{ClinVar data analysis \\ MATERIALS AND METHODS}

A custom report was generated by ClinVar on January 6, 2016, to identify all alleles (measure_ids) in ClinVar with submissions from at least two of the four participating laboratories (Ambry, GeneDx, LMM, and UCGSL) and to report the interpretations from all participating laboratories for each identified allele. For each allele, interpretations were compared to determine concordance or discordance; if discordant, the level of discordance was assigned. For variants with more than two interpretation terms submitted, the two most discordant terms were used to assign the level of discordance. For example, a variant with a pathogenic, likely pathogenic, and uncertain significance interpretation would be categorized by the pathogenic versus uncertain significance difference. Metrics were calculated for all four laboratories in aggregate as well as for each pairwise comparison of the four laboratories to facilitate identification of variants for reassessment.

\section{Resolution process}

Each laboratory compared its interpretations in ClinVar to their current internal interpretations to determine whether the difference was resolved by a more recent reassessment not yet represented in ClinVar. Next, each pair of laboratories identified a subset of variants with interpretation differences for reassessment (242 variants of the total 724 variants with interpretation differences), prioritizing variants with $\mathrm{P} /$ $L P$ versus $L B / B$ differences and genes and/or disease areas with a higher frequency of interpretation differences, followed by additional medically significant differences. After variants had been identified for reassessment, participating laboratories shared internal data, discussed initial classification rationale, and categorized the reason for the initial interpretation difference when it was apparent (e.g., differences in classifications rules or internal data). After discussion, laboratories independently reassessed variants with the ACMG-AMP guidelines and, if appropriate, reclassified the variant in their own database. Laboratories continued discussions for variants with interpretations that remained different after internal reassessment, allowing a consensus to be achieved for an additional set of discordant variants. For variants remaining discordant, any unique ACMG-AMP criteria applied by one laboratory but not 
the other participating laboratory were tracked. For statistical comparisons, a Fisher's exact test was used.

\section{RESULTS}

\section{Initial concordance data}

As of January 2016, more than 49,000 unique variants had been submitted to ClinVar by at least one of the four participating laboratories (Ambry, GeneDx, LMM, and UCGSL). Comparison of data in ClinVar identified 6,169 variants from 308 genes interpreted by at least two of the four participating laboratories. This large number of variants interpreted by at least two participating clinical laboratories facilitated comparison of interpretations and potential resolution of differences.

Of the 6,169 shared variants (Figure 1a), 88.3\% of interpretations between laboratories were concordant, with $62.3 \%$ (3,845 variants) in exact agreement and $26.0 \%$ (1,600 variants) differing only in confidence levels ( $\mathrm{P}$ versus LP and LB versus $B)$. Because confidence differences are unlikely to impact clinical care or management, they were not considered discordant for this project and are included in concordant counts. The remaining $11.7 \%$ (724) of shared variants had discordant interpretations and were categorized by the level of discordance; $3.5 \%$ (216 variants) of all shared variants were medically significant differences (MSDs), pathogenic (P/LP) versus other (VUS/LB/B), which are most likely to impact medical management. However, the majority of MSDs (94.4\%) were $\mathrm{P} / \mathrm{LP}$ versus VUS differences, and only 12 variants of this set (5.6\%) had $\mathrm{P} / \mathrm{LP}$ versus $\mathrm{LB} / \mathrm{B}$ differences. The remaining $8.2 \%$ (508 variants) of all shared variants were VUS versus benign $(\mathrm{LB} / \mathrm{B})$ differences, which are less likely to affect medical management but may lead to differences in counseling time and medical management. Analysis of concordance and discordance by variant type showed the highest concordance (97.0\%; 479/494) for interpretation of predicted null variants (nonsense, frameshift, and $\pm 1,2$ splice site), with these differences accounting for only $0.8 \%(4 / 508)$ of all VUS versus LB/B differences and 5.1\% (11/216) of all MSDs. Interpretations of missense variants were $84.6 \%$ concordant $(3,053 / 3,607)$; however, as expected, missense differences accounted for the highest percent of both VUS versus LB/B differences $(70.9 \% ; 360 / 508)$ and MSDs $(89.8 \% ; 194 / 216)$.

The 724 variants with interpretation differences were from 148 genes. To determine trends by disease area, genes were categorized into four categories (cardiovascular, hereditary cancer, neurologic, and other); the proportion of MSDs and other differences are presented in the first column for each disease area ("Total") in Figure 2. See Supplementary Table S1 online for pre- and postresolution concordance rates for each disease area. The neurologic disease area had the lowest initial concordance (81.2\%), with the majority of differences being VUS versus $\mathrm{LB} / \mathrm{B}$ compared to MSDs (17.8\% and $1.0 \%$ of all neurologic variants, respectively). Cardiovascular was the only disease area with more initial MSDs than VUS versus LB/B differences (5.9 and $5.2 \%$ of all cardiovascular variants, respectively). A summary of each of the 148 genes with interpretation differences, including the number of shared variants and percent concordant versus discordant, is provided in Supplementary Table S2 online.

\section{Resolution of interpretation differences}

To facilitate resolution of interpretation differences between laboratories, each pairwise combination of the four participating laboratories was compared to determine the number of interpretation differences from each pair of laboratories (Supplementary Table S3 online). Laboratory pairs with a higher number of total interpretation differences selected variants for reassessment based on the level of discordance (prioritizing variants with medically significant differences, especially those with $\mathrm{P} / \mathrm{LP}$ versus LB/B differences) and by disease area, with cardiovascular reassessments prioritized by genes with multiple interpretation differences (MYBPC3, GLA, ACTC1, MYL2, MYL3, TPM1, RAF1) and hereditary cancer reassessments prioritized by variants in high-risk hereditary breast and ovarian cancer genes or genes with National Comprehensive Cancer Network (NCCN) criteria for management (BRCA1, $B R C A 2, C D H 1, P T E N, T P 53)$. Laboratory pairs with lower total interpretation differences prioritized variants for reassessments based simply on the level of discordance. In total, laboratories reassessed 242 variants with interpretation differences (116 variants with MSDs and 126 variants with other differences) to determine whether sharing internal data and applying the ACMG-AMP criteria and classification rules could resolve the different interpretations (Figure 3).

For the 116 reassessed variants with MSDs, 79.3\% (92 variants) were resolved by reassessment, data sharing, and discussion, with $19.8 \%$ (23 variants) resolved as P/LP, 58.6\% (68 variants) resolved as VUS, and $0.9 \%$ (1 variant) resolved as LB. For the 126 reassessed variants with VUS versus LB/B differences, $94.4 \%$ (119 variants) were resolved, with $16.7 \%$ (21 variants) resolved as VUS and $77.8 \%$ (98 variants) resolved as $\mathrm{LB} / \mathrm{B}$. In total, 211 of 242 reassessed variants $(87.2 \%)$ were resolved. Comparing disease areas (Figure 2), laboratories had a significantly higher resolution rate for cardiovascular variants than hereditary cancer variants $(P=0.00059)$ and neurologic variants $(P=0.027)$; no significant differences were observed between hereditary cancer and neurologic variants (Figure 2). The difference between cardiovascular and hereditary cancer resolution rates is due to a significantly higher resolution rate specifically for cardiovascular MSDs (74/77) versus hereditary cancer MSDs $(17 / 32 ; P=0.0001)$. No significant differences in the resolution of VUS versus LB/B interpretation differences were observed between disease areas. See Supplementary Table S4 online for a full list of resolved variants. Because classifications may change over time with new evidence, please check ClinVar for the most up-to-date classifications.

For a subset of variants with resolved interpretation differences (100 variants), the reasons for the initial interpretation differences and whether reassessment with ACMG/AMP guidelines and/or sharing internal evidence impacted the reclassification were documented (Figure 4). For each variant, laboratories decided which of the predefined categories best 
a

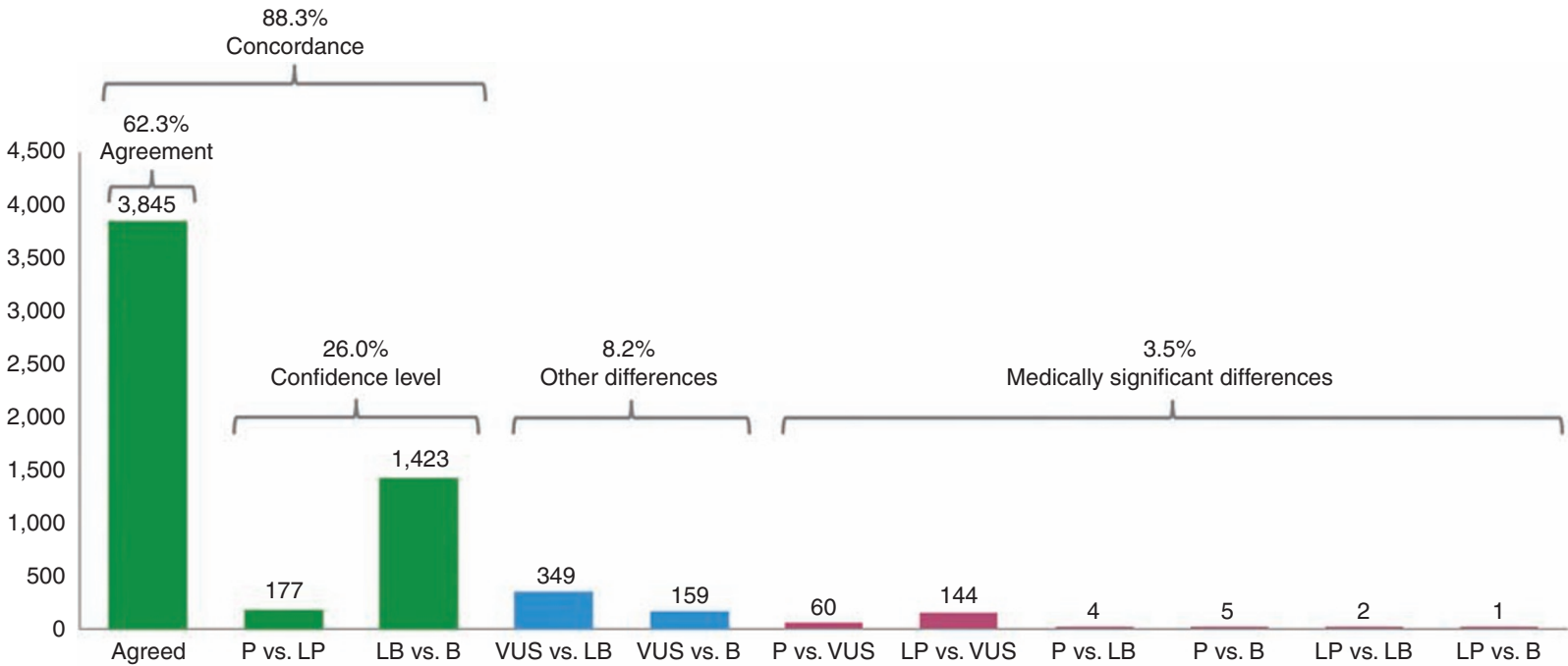

b

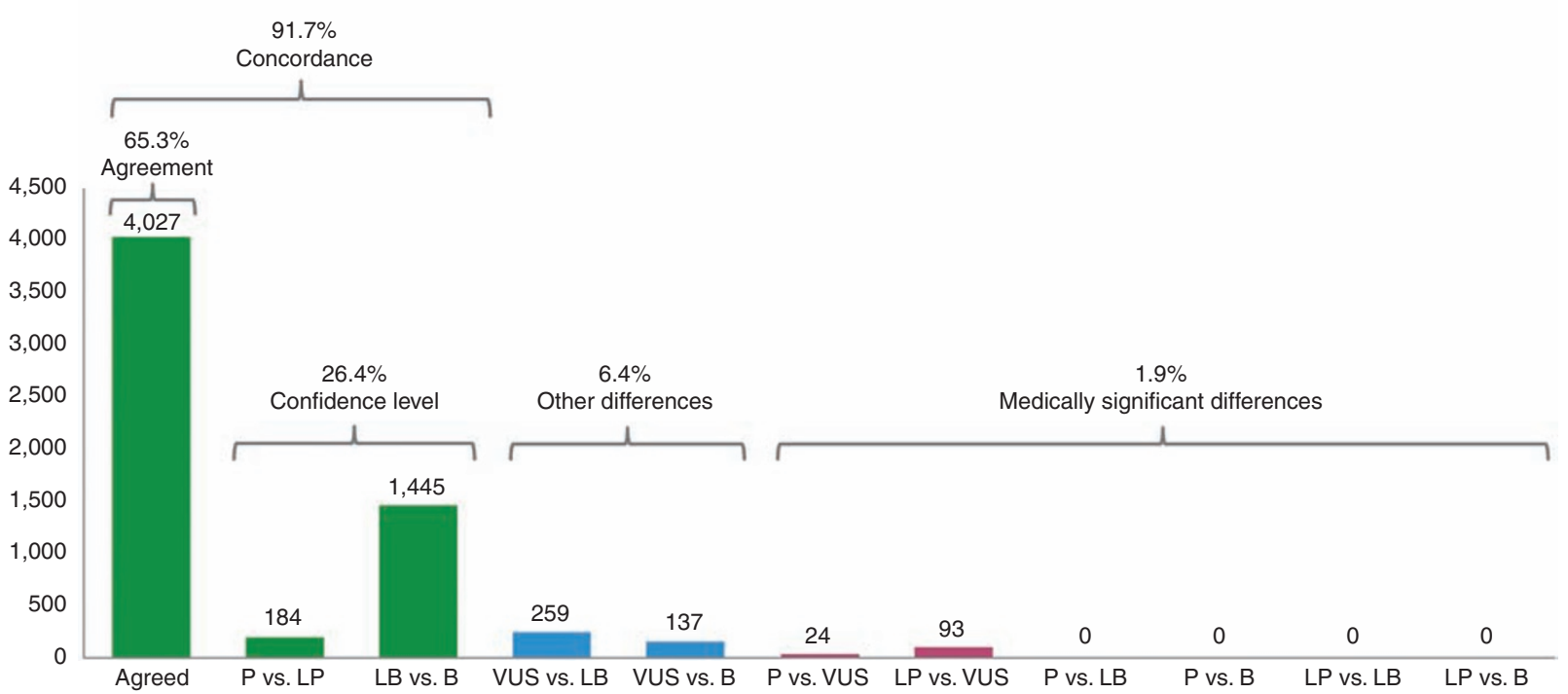

Figure 1 Distribution of variant interpretation differences between four clinical laboratories. (a) Interpretation comparison of data in ClinVar (as of January 1, 2016) before resolution efforts. (b) Interpretation comparison after reassessing 33\% (242/724) of shared variants with interpretation differences.

explained the initial difference in interpretation. Reassessment of older variant interpretations with updated classification criteria, consistent with ACMG-AMP guidelines, resolved $36 \%$ of variants (Figure 4, red shading). Sharing internal data facilitated resolution of $33 \%$ of resolved variants (blue shading), with sharing segregation data (10\%) and co-occurrence data (9\%) facilitating the largest percent of variants. Differences in the use or weighting of public data accounted for $14 \%$ of interpretation differences (purple shading), including benign/likely benign thresholds (9\%) and different data sources (5\%). Finally, for $17 \%$ of resolved variants, the interpretation differences were found to have already been resolved, but the new interpretations were not yet submitted to ClinVar.

\section{Persistent interpretation differences}

After reassessment and data sharing, laboratories were unable to reach consensus for $12.8 \%$ ( 31 variants) of the 242 reassessed variants, of which 24 are unresolved MSDs and 7 are unresolved other differences (Supplementary Table S5 online). For all unresolved differences, reassessments from the submitting laboratories were compared to determine the unique ACMGAMP criteria applied that accounted for the different clinical interpretations (Table 1). The unique ACMG-AMP criteria were then grouped into evidence categories, as shown in Figure 1 of the paper by Richards et al., ${ }^{8}$ to determine whether specific categories of evidence were more likely to be differently applied than others. For the persistent MSDs, 50\% (12 variants) were impacted by differences in the application of benign criteria, such as minor allele frequency data (BS1), observation in unaffected adults (BS2), and functional studies showing no deleterious effect (BS3). The remaining persistent MSDs were due to different application of pathogenic criteria, with evidence in the functional data category impacting the highest percent of variants. For the seven variants with unresolved VUS versus LB/B 


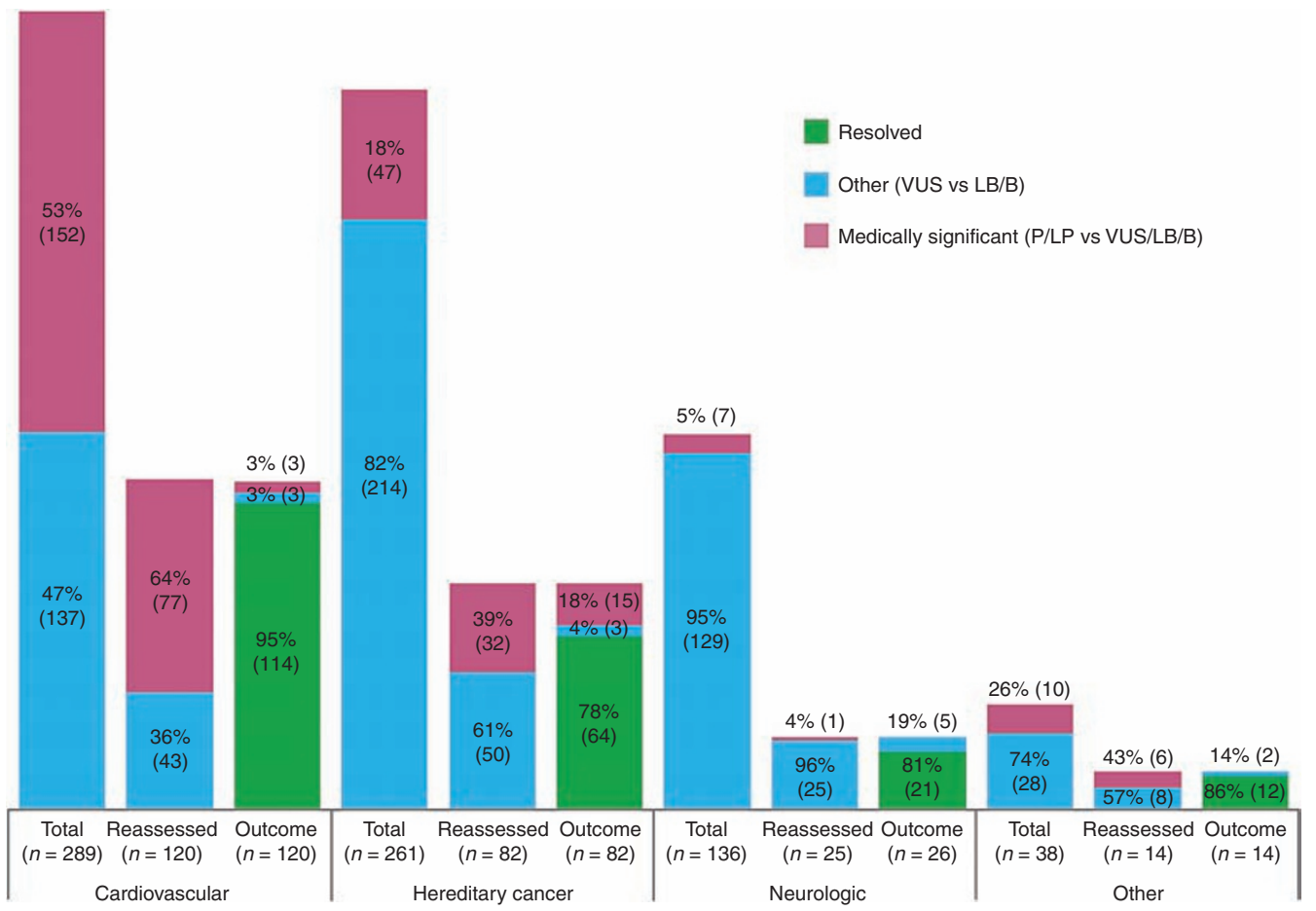

Figure 2 Distribution of interpretation differences and resolution outcome per disease area. Distribution of medically significant (P/LP versus VUS/ LB/B) and other (VUS versus LB/B) differences within each disease area for all initial differences ("Total"), variants reassessed by laboratories ("Reassessed"), and final outcome for reassessed variants, including proportion resolved ("Outcome").

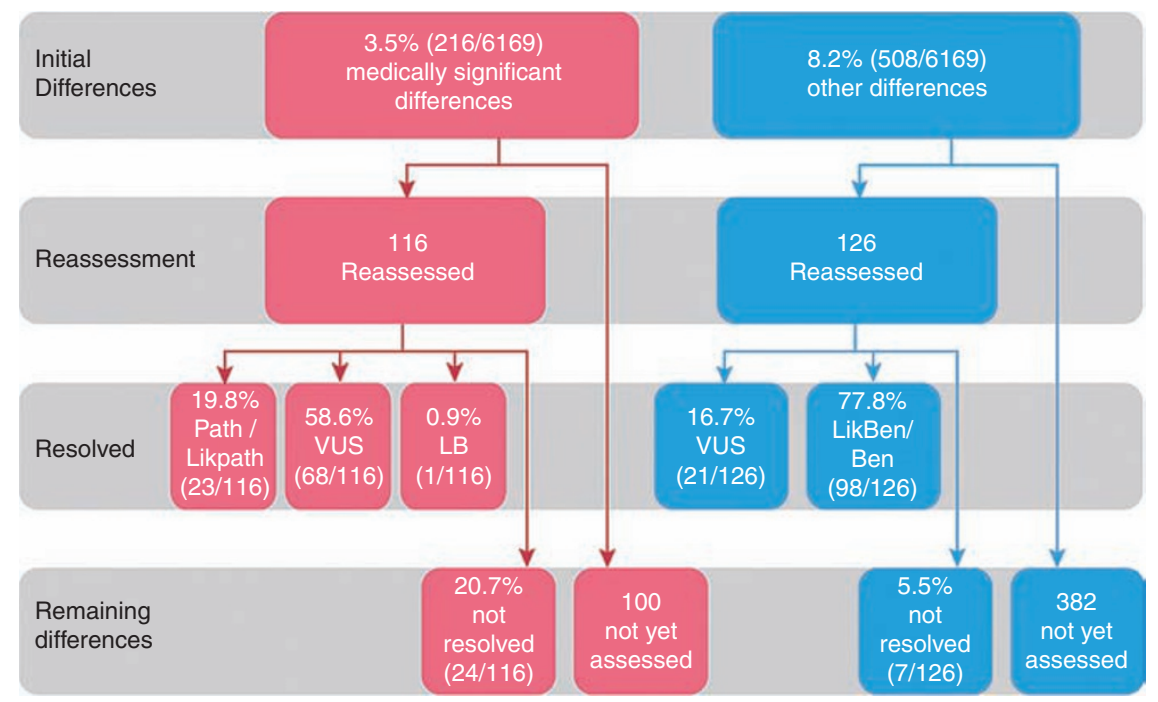

Figure 3 Flowchart and outcome of variant resolution efforts.

differences (Table 1), the most frequent differentially applied ACMG-AMP criteria was minor allele frequency data (BS1). In total, across both discordant types, evidence in the functional data category (48\%) and population data category (45\%) contributed to the highest number of persistent interpretation differences.

Additionally, even though interpretations remained discordant, for seven variants reassessment reduced the level of discordance from a two-step difference (P/LP versus $\mathrm{B} / \mathrm{LB}$ ) to a one-step difference (P/LP versus VUS or VUS versus $\mathrm{LB} / \mathrm{B}$ ). These variants are highlighted in gray in Supplementary Table S5 online.

\section{DISCUSSION}

Data sharing through ClinVar offers a unique opportunity to identify classification differences between laboratories and to collaborate to resolve these differences. By submitting variant interpretations to ClinVar and working together, four clinical 


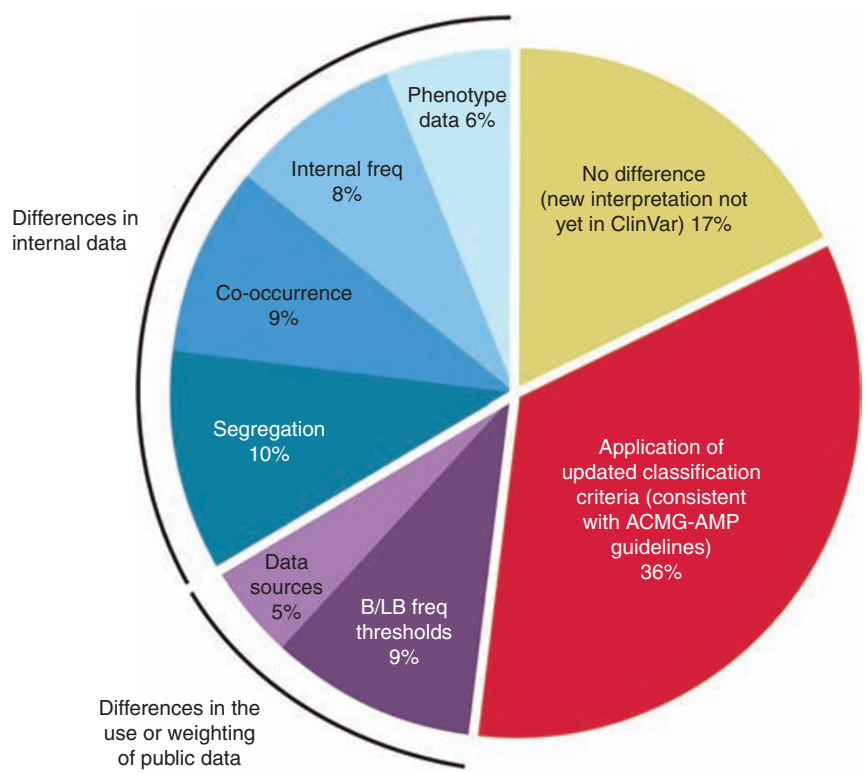

Figure 4 Basis of initial interpretation differences for resolved variants. More than half of the initial interpretation differences were resolved simply because the re-interpretation had already been completed but was not yet submitted to ClinVar (17\%, yellow shading) or by reassessing an old variant interpretation with the laboratory's updated classification criteria, consistent with ACMG-AMP guidelines (36\%, red shading). Differences in the use or weighting of public data accounted for $14 \%$ of interpretation differences (purple shading), including benign/likely benign thresholds (9\%), and different data sources (5\%). Differences in internal data accounted for $33 \%$ of interpretation differences (blue shading), including segregation data $(10 \%)$, co-occurrence data $(9 \%)$, internal proband frequency $(8 \%)$, and detailed phenotype data (6\%).

laboratories were able to resolve differences in $87.2 \%$ of initially discordant variants that were reviewed (211/242 variants). With only $33.4 \%$ of all variants with interpretation differences reviewed so far (242/724 variants), participating laboratories have already increased their overall concordance rate from 88.3 to $91.7 \%$ (Figure 1b), indicating that sharing internal evidence and classification rationales is critical for moving toward more consistent variant interpretations. This concordance rate is higher than that in previous reports because the scope was limited to a set of four clinical laboratories, as opposed to a report of $77 \%$ concordance between all ClinVar submitters of a set of variants ${ }^{6}$ and because participating laboratories shared all evidence such that interpretations are based on the same set of evidence, as opposed to a report of only $40 \%$ concordance between laboratories when internal data were not shared. ${ }^{5}$ Additionally, both the initial concordance $(88.3 \%)$ and the resolution rate (87.2\%) were higher than reported by the nine sites in the CSER program (56.6 and $72.5 \%$, respectively) ${ }^{7}$ because the majority of selected CSER variants were outside the scope of variants and genes encountered by the CSER sites during routine clinical testing, suggesting that expertise in disease areas may contribute to increased concordance.

Implementation of the evidence-based 2015 ACMG-AMP guidelines for sequence variant interpretation has alleviated some burden of differences due to classification algorithms.
Thirty-six percent of resolved variants were initially discordant due to differences in each laboratory's internal classification method used at the time of initial assessment, but the interpretation differences were resolved by reassessment with laboratories' current criteria, consistent with ACMGAMP guidelines. However, implementation of the guidelines still requires professional judgment in deciding which evidence types are met $-12.8 \%$ of variants reassessed with the guidelines were unable to be resolved. It is important to note that for the persistent interpretation differences, laboratories had access to the same data, including shared internal data; however, the interpretation of that data in the context of meeting specific ACMG-AMP criteria differed. The majority of persistent interpretation differences were due to differences in the application of functional data (48\%) and population data (45\%), both of which require gene- or disease-specific knowledge. Differences in the application of these criteria suggest that further gene specification of the ACMG-AMP guidelines by experts, such as providing specific allele frequency cutoffs and indicating which functional assays are considered well established, could increase concordance. Specifically, comparison of the reassessment outcome between four disease areas indicates that genes in the inherited cancer disease area would greatly benefit from ACMG-AMP specification because this disease area had the lowest rate of resolution (78\%) and highest rate of persistent medically significant interpretation differences (Figure 2; Supplementary Table S1 online).

Recent publications have provided feedback and recommendations for the ACMG-AMP guidelines. ${ }^{710,11}$ ClinGen disease-specific working groups and the ClinGen Sequence Variant Interpretation working group are focused on specifying the ACMG-AMP guidelines for diseases of interest, including minor allele frequency thresholds and gene-specific functional assay and domain data, as well as providing further quantitative guidance regarding individual criteria such as segregation data, population data, and case data. These recommendations will become public once completed and approved by ClinGen.

Our analyses demonstrated that resolution of $33 \%$ of variant interpretation differences benefited from sharing internal data. Such data have typically been inaccessible except for the small subset of data published in the literature, often many years after it was identified. This leads to important information that could affect patient diagnoses being unavailable to individuals who could benefit. However, through submission to ClinVar, laboratories can more quickly share these data as they accumulate, to the benefit of other laboratories and the patients they serve. It should be noted that some, but not all, clinical laboratories provide detailed supporting evidence in ClinVar, in the form of citations, text-based interpretation summaries, and case-level observations. Still, even for laboratories that have not yet shared this detail, ClinVar submissions at least identify that a laboratory has observed the variant and prompts further collaborative investigations and data sharing when patient treatment decisions could be impacted. 
Table 1 Unique ACMG/AMP criteria applied contributing to persistent interpretation differences grouped into evidence categories

\begin{tabular}{|c|c|c|c|c|c|}
\hline $\begin{array}{l}\text { ACMG-AMP evidence } \\
\text { category }\end{array}$ & $\begin{array}{c}\% \text { Persistent } \\
\text { differences impacted }\end{array}$ & ACMG-AMP rule & Description & $\begin{array}{l}\text { No. P/LP } \\
\text { versus VUS/ } \\
\text { LB/B (\%) }\end{array}$ & $\begin{array}{c}\text { No. VUS } \\
\text { versus } \\
\text { LB/B (\%) }\end{array}$ \\
\hline \multirow[t]{4}{*}{ Functional data } & \multirow[t]{4}{*}{$48 \%$} & PS3 & $\begin{array}{l}\text { Well-established functional studies show a } \\
\text { deleterious effect }\end{array}$ & $7(29 \%)$ & 0 \\
\hline & & PM1 & $\begin{array}{l}\text { Mutational hot spot or well-studied function } \\
\text { domain without benign variation }\end{array}$ & $5(21 \%)$ & 0 \\
\hline & & PP2 & $\begin{array}{l}\text { Missense in gene with low rate of benign } \\
\text { missense variants and path missense common }\end{array}$ & $1(4 \%)$ & 0 \\
\hline & & BS3 & $\begin{array}{l}\text { Well-established functional studies show no } \\
\text { deleterious effect }\end{array}$ & $2(8 \%)$ & 0 \\
\hline \multirow[t]{3}{*}{ Population data } & \multirow[t]{3}{*}{$45 \%$} & BS1 & Minor allele frequency is too high for disorder & $4(17 \%)$ & $4(57 \%)$ \\
\hline & & BS2 & $\begin{array}{l}\text { Observation in controls inconsistent with disease } \\
\text { penetrance }\end{array}$ & $3(13 \%)$ & $1(14 \%)$ \\
\hline & & PS4 & $\begin{array}{l}\text { Prevalence in affected statistically increased over } \\
\text { controls }\end{array}$ & $2(8 \%)$ & 0 \\
\hline \multirow[t]{2}{*}{ Other databases } & \multirow[t]{2}{*}{$29 \%$} & PP5 & Reputable source $=$ pathogenic & $8(33 \%)$ & 0 \\
\hline & & BP6 & Reputable source $=$ benign & $1(4 \%)$ & 0 \\
\hline \multirow[t]{3}{*}{$\begin{array}{l}\text { Computational and predictive } \\
\text { data }\end{array}$} & \multirow[t]{3}{*}{$16 \%$} & BP4 & $\begin{array}{l}\text { Multiple lines of computational evidence suggest } \\
\text { no impact on gene product }\end{array}$ & $1(4 \%)$ & $1(14 \%)$ \\
\hline & & PM5 & $\begin{array}{l}\text { Novel missense change at a residue where a } \\
\text { different path missense change has been seen }\end{array}$ & $2(8 \%)$ & 0 \\
\hline & & PP3 & $\begin{array}{l}\text { Multiple lines of computational evidence suggest } \\
\text { a deleterious effect on gene product }\end{array}$ & $1(4 \%)$ & 0 \\
\hline \multirow[t]{2}{*}{ Other data } & \multirow[t]{2}{*}{$13 \%$} & PP4 & Patient's phenotype or FH highly specific for gene & $3(13 \%)$ & 0 \\
\hline & & BP5 & Found in case with an alternative cause & 0 & $1(14 \%)$ \\
\hline \multirow[t]{2}{*}{ Segregation data } & \multirow[t]{2}{*}{$6 \%$} & PP1 & $\begin{array}{l}\text { Cosegregation with disease in multiple affected } \\
\text { family members }\end{array}$ & 0 & $1(14 \%)$ \\
\hline & & BS4 & Nonsegregation with disease & $1(4 \%)$ & 0 \\
\hline Allelic data & $3 \%$ & PM3 & $\begin{array}{l}\text { For recessive disorders, detected in trans with a } \\
\text { pathogenic variant }\end{array}$ & $1(4 \%)$ & 0 \\
\hline
\end{tabular}

ACMG-AMP benign criteria codes begin with B and pathogenic criteria codes begin with P. ACMG-AMP, American College of Medical Genetics and Genomics-Association for Molecular Pathology; LB/B, likely benign/benign; P/LP, pathogenic/likely pathogenic; VUS, variants of uncertain significance; FH, family history.

More than one-third of the variant interpretation differences were resolved simply by one laboratory reassessing an old variant interpretation with the laboratory's current criteria, consistent with ACMG-AMP guidelines. Most clinical laboratories reassess variants when they are observed in an additional case or at the request of providers. Thus, for many remaining interpretation differences not yet reviewed, routine clinical laboratory reassessment with current criteria will resolve the difference regardless of sharing internal evidence. Although these recommendations will benefit some variants, many variants identified in Mendelian disease testing are extremely rare, ${ }^{1}$ underscored by the observation that $88 \%$ of the 49,734 unique variants in this study were submitted by only one of the four laboratories. In addition, analysis of the sequencing data from 60,706 individuals in the Exome Aggregation Consortium (ExAC) found that $99 \%$ of identified high-quality variants have an allele frequency less than $1 \%$ and that $54 \%$ of identified highquality ExAC variants are only seen once in the entire data set. ${ }^{12}$ These findings further emphasize that the vast majority of variants identified by clinical testing laboratories are incredibly rare or even private and thus are unlikely to be regularly reassessed or potentially ever interpreted again. Therefore, it is critical for laboratories to share their observations and identify differences, which may be the only prompt for reassessment.

Differences in interpretation of data are not unique to clinical genetic laboratories; other health-care providers are also subject to discordance in professional interpretation. When pathologists' interpretations of individual breast biopsies were compared with consensus-derived reference diagnosis, the overall concordance was only $75.3 \%{ }^{13}$ Similarly, radiologists were only $83 \%$ concordant in assessing dense versus nondense status of breast mammograms. ${ }^{14}$ These findings suggest that a consensus-based approach of multiple experts collaborating on a diagnosis may be necessary to improve interpretation. Similar approaches have been seen in rare-disease genetics with the formation of international expert consortia that can provide consensus interpretations of variants, such as the International Society for Gastrointestinal Hereditary Tumours (InSiGHT) and the Evidence-based Network for the Interpretation of Germline Mutation Alleles (ENIGMA). ${ }^{15,16}$ These diseasefocused efforts not only apply expert interpretation but also can organize data-gathering efforts and spur research studies that 
can lead to a better understanding of genetic variation in their respective domains. For example, InSiGHT reclassified 54\% of variants submitted with uncertain significance interpretations as pathogenic or likely pathogenic. ${ }^{15}$ To expand these efforts, ClinGen has formed numerous disease expert groups (https:// www.clinicalgenome.org/working-groups/clinical-domain/) and continues to solicit further applications from groups to be considered as expert panels (three-star level) with respect to their ClinVar submission status (http://www.ncbi.nlm.nih.gov/ clinvar/docs/review_guidelines/). Once a three- or four-star variant interpretation is submitted to ClinVar from an expert panel, it will override submissions of lower review status to continually improve the reliability of public databases for use in diagnosis. However, these efforts benefit heavily from single laboratory submissions to identify variants that should be subject to expert review.

Laboratories did not reassess all 724 variants with interpretation differences because the goals of this pilot project were to determine whether sharing internal data and implementing the ACMG-AMP guidelines could resolve differences and to create a framework for addressing all differences in ClinVar. In continuing to reassess the remaining variants with interpretation differences between these four clinical laboratories, further emphasis will be placed on engaging additional ClinVar submitters. To promote more consistent variant interpretations in this field, participation from all submitters on a variant with conflicting interpretations is necessary to move more variants from a single-star review status to a two-star review status, indicating that all submitted interpretations of a variant are concordant. This process will be overseen by ClinGen's Sequence Variant Inter-Laboratory Resolution working group, which will work on addressing all interpretation differences in ClinVar from all submitters. The lessons learned from this pilot project will guide the support and infrastructure needed to identify differences and facilitate sharing of internal data and classification rationale. For instance, in this study, we found that $36 \%$ of interpretation differences were resolved simply by reassessing an older interpretation with laboratories' updated classification criteria. Given the time commitment to this project (an estimated 1-2 hours per variant per laboratory), future resolution will begin by encouraging laboratories with older interpretations or outlier interpretations to first reassess the variant with current guidelines, which would minimize the total time commitment for all laboratories.

In conclusion, these results demonstrate that clinical interpretations from these four clinical laboratories are now concordant for $91.5 \%$ of shared variants in ClinVar, with only $2 \%$ of variant interpretation differences considered medically significant. Further specification regarding genespecific criteria and expert guidance on the relevance of conflicting pathogenic and benign criteria may further facilitate resolution of interpretation differences with ACMG-AMP guidelines. Given that the interpretation of variants for their role in disease requires expert opinion and subjective review of scientific evidence and medical data, complete concordance is not expected. However, increased training and guidance regarding the application of the ACMG-AMP criteria and ensuring full sharing of evidence and classification rationales are critical to move toward more consistent variant interpretations, which will improve the care of patients with, or at risk for, genetic disorders.

\section{SUPPLEMENTARY MATERIAL}

Supplementary material is linked to the online version of the paper at http://www.nature.com/gim

\section{ACKNOWLEDGMENTS}

Research reported in this publication was supported by the National Human Genome Research Institute (NHGRI) in conjunction with funding from the Eunice Kennedy Shriver National Institute of Child Health and Human Development (NICHD) under award U41HG006834. The content is solely the responsibility of the authors and does not necessarily represent the official views of the National Institutes of Health. We thank all ClinVar submitters for sharing their data, and Jim Carver and Jessica Burgess for creating the figures.

\section{DISCLOSURE}

All authors are clinical service providers and are employed by laboratories that offer fee-based clinical sequencing. This employment is noted in the author affiliations. The authors declare no additional conflicts of interest beyond their employment affiliation.

\section{REFERENCES}

1. Rehm HL, Berg JS, Brooks LD, et al.; ClinGen. ClinGen-the clinical genome resource. N Engl J Med 2015;372:2235-2242.

2. Landrum MJ, Lee JM, Benson M, et al. ClinVar: public archive of interpretations of clinically relevant variants. Nucleic Acids Res 2016;44(D1):D862-D868.

3. Amendola LM, Dorschner MO, Robertson PD, et al. Actionable exomic incidental findings in 6503 participants: challenges of variant classification. Genome Res 2015;25:305-315.

4. Yorczyk A, Robinson LS, Ross TS. Use of panel tests in place of single gene tests in the cancer genetics clinic. Clin Genet 2015;88:278-282.

5. Pepin MG, Murray ML, Bailey S, Leistritz-Kessler D, Schwarze U, Byers PH. The challenge of comprehensive and consistent sequence variant interpretation between clinical laboratories. Genet Med 2016;18:20-24.

6. Balmaña J, Digiovanni L, Gaddam P, et al. Conflicting interpretation of genetic variants and cancer risk by commercial laboratories as assessed by the prospective registry of multiplex testing. J Clin Onco/ 2016;34:4071-4078.

7. Amendola LM, Jarvik GP, Leo MC, et al. Performance of ACMG-AMP variantinterpretation guidelines among nine laboratories in the Clinical Sequencing Exploratory Research Consortium. Am J Hum Genet 2016;99:247.

8. Richards S, Aziz N, Bale S, et al.; ACMG Laboratory Quality Assurance Committee. Standards and guidelines for the interpretation of sequence variants: a joint consensus recommendation of the American College of Medical Genetics and Genomics and the Association for Molecular Pathology. Genet Med 2015;17:405-424.

9. Green RC, Goddard KA, Jarvik GP, et al.; CSER Consortium. Clinical Sequencing Exploratory Research Consortium: Accelerating evidence-based practice of genomic medicine. Am J Hum Genet 2016;98:1051-1066.

10. Maxwell KN, Hart SN, Vijai J, et al. Evaluation of ACMG-guideline-based variant classification of cancer susceptibility and non-cancer-associated genes in families affected by breast cancer. Am J Hum Genet 2016;98:801-817. 
11. Jarvik GP, Browning BL. Consideration of cosegregation in the pathogenicity classification of genomic variants. Am J Hum Genet 2016;98:1077-1081.

12. Lek M, Karczewski KJ, Minikel EV, et al.; Exome Aggregation Consortium. Analysis of protein-coding genetic variation in 60,706 humans. Nature 2016;536:285-291.

13. Elmore JG, Longton GM, Carney PA, et al. Diagnostic concordance among pathologists interpreting breast biopsy specimens. JAMA 2015;313:1122-1132.

14. Sprague BL, Conant EF, Onega T, et al.; PROSPR Consortium. Variation in mammographic breast density assessments among radiologists in clinical practice: a multicenter observational study. Ann Intern Med 2016;165:457464.

15. Thompson BA, Spurdle AB, Plazzer JP, et al.; InSiGHT. Application of a 5-tiered scheme for standardized classification of 2,360 unique mismatch repair gene variants in the InSiGHT locus-specific database. Nat Genet 2014;46:107-115.

16. Spurdle AB, Healey $S$, Devereau A, et al.; ENIGMA. ENIGMA-evidence-based network for the interpretation of germline mutant alleles: an international initiative to evaluate risk and clinical significance associated with sequence variation in BRCA1 and BRCA2 genes. Hum Mutat 2012;33:2-7. 